\title{
关于钢制结构表面处理控制要点
}

\section{张海奎 李春梅}

北京兴油工程项目管理有限公司，北京 100089

中国石油化工股份有限公司西北油田分公司，新疆乌鲁木齐 830011

DOI:10.33142/ec.v2i2.148

[摘要]钢在结构在现代石油工程中得到应用广泛, 但腐蚀问题已经成为影响钢结构产品质量的最重要的因素之一, 本文 主要讨论钢制结构表面处理过程中需要控制的主要要点。

[关键词]腐蚀; 表面处理; 含盐量

\section{The Control Points of Surface Treatment of Steel Structure}

\section{ZHANG Haikui, LI Chunmei}

\section{Beijing Xingyou Engineering Project Management Co.,Ltd.Beijing,China,100089}

\section{Northwest Oil Field Branch of Sinopec Corp.,Urumqi,Xinjiang,China,830011}

Abstract: Steel structure is widely used in modern petrol engineering,but corrosion problem has became one of the key factors influencing the quality of steel structural products. This paper discusses the main control points in the process of steel structure surface treatment.

Keywords: Corrosion; Surface treatment;Salinity

\section{引言}

随着石油工业和社会发展，钢制材料得到广泛运用，但受使用环境影响，金属腐蚀已成为影响材料结构及质量安 全最重要的因素之一, 造成严重的经济损失和潜在的安全风险, 因此为确保材料使用安全以及可持续发展, 在石油开采、 运输、炼化、生产过程中, 我们经常需重新对钢制结构进行除锈、喷漆防腐处理。但由于行业、国家以及运行环境不 同, 处理标准不统一, 导致现场作业时目标不明确, 为了方便施工及监督管理, 现对钢制结构表面处理进行讨论分析, 梳理关键控制点。

1 腐蚀分类

为了明确腐蚀类别，根据腐蚀环境不同，IS09223 将腐蚀分为大气、水、土壤腐蚀三大类，因此在钢结构涂装之前， 设计单位需要根据项目环境及工作条件, 确定相应的涂装体系, 腐蚀环境的分类和定义见表 $1 、 2$ 。

$$
\text { 表 } 1 \text { - 大气环境腐蚀性分类和典型环境案例 }
$$

\begin{tabular}{|c|c|c|c|c|c|c|}
\hline \multirow{3}{*}{ 腐蚀级别 } & \multicolumn{4}{|c|}{ 单位面积上质量和厚度损失（经第1 年暴露后） } & \multicolumn{2}{|c|}{ 温性气候下的典型环境案例（仅供参考） } \\
\hline & \multicolumn{2}{|c|}{ 低碳钢 } & \multicolumn{2}{|c|}{ 锌 } & \multirow[b]{2}{*}{ 外部 } & \multirow[b]{2}{*}{ 内部 } \\
\hline & $\begin{array}{c}\text { 质量损失 } \\
/ \mathrm{g} \cdot \mathrm{m}-2\end{array}$ & 质量损失/um & $\begin{array}{c}\text { 质量损失 } \\
/ \mathrm{g} \bullet \mathrm{m}-2\end{array}$ & $\begin{array}{l}\text { 质量损 } \\
\text { 失/um }\end{array}$ & & \\
\hline C1很低 & $\leqslant 10$ & $\leqslant 1.3$ & $\leqslant 0.7$ & 0.1 & & $\begin{array}{l}\text { 加热的建筑物内部, 空 } \\
\text { 气洁净, 如办公室、商 } \\
\text { 店、学校和宾馆等 }\end{array}$ \\
\hline C2低 & $>100-200$ & $>1.3-25$ & $>0.7-5$ & $>0.1-0.7$ & $\begin{array}{l}\text { 低污染水平的大 } \\
\text { 气, 大部分是乡村 } \\
\text { 地带 }\end{array}$ & $\begin{array}{l}\text { 冷凝有奇能发生的未 } \\
\text { 加热的建筑（如库房, } \\
\text { 体育馆等）。 }\end{array}$ \\
\hline C3中 & $>200-300$ & $>25-50$ & $>5-15$ & $>0.7-2.1$ & $\begin{array}{l}\text { 城市和工业大气, } \\
\text { 中等的二氧化硫污 } \\
\text { 染以及低盐度沿海 } \\
\text { 区域 }\end{array}$ & $\begin{array}{l}\text { 高湿度和有些空气污 } \\
\text { 染的生产厂房内, 如食 } \\
\text { 品加工厂、洗衣场、酒 } \\
\text { 厂乳制品工厂等。 }\end{array}$ \\
\hline C4高 & $>400-650$ & $>50-80$ & $>15-30$ & $>2.1-4.2$ & $\begin{array}{l}\text { 中等含盐度的工业 } \\
\text { 区和沿海区域 }\end{array}$ & $\begin{array}{l}\text { 化工厂、游泳池、沿海 } \\
\text { 船舶和造船厂等。 }\end{array}$ \\
\hline
\end{tabular}




\begin{tabular}{|c|c|c|c|c|c|c|}
\hline C5很高 & $>650-1500$ & $>80-200$ & $>30-60$ & $>4.2-8.4$ & $\begin{array}{l}\text { 高湿度和恶劣大气 } \\
\text { 的工业区域和高含 } \\
\text { 盐度的沿海区域。 }\end{array}$ & $\begin{array}{l}\text { 冷凝和高污染持续发 } \\
\text { 生 和存在 的建 筑和 区 } \\
\text { 域。 }\end{array}$ \\
\hline CX极端 & $\begin{array}{l}>1500^{-} \\
5500\end{array}$ & $>200-700$ & $>60-180$ & $>8.4-25$ & $\begin{array}{l}\text { 具有高含盐度的海 } \\
\text { 上区域以及具有极 } \\
\text { 高湿度和侵蚀性大 } \\
\text { 气的热带亚热带工 } \\
\text { 业区域。 }\end{array}$ & $\begin{array}{l}\text { 具有极高湿度和侵蚀 } \\
\text { 性大气的工业区域。 }\end{array}$ \\
\hline
\end{tabular}

CX 涵盖了不同的极端环境。一个特定的极端环境是由 IS0 12944-9 所涵盖的离岸环境。其它的极端环境没有包含 在 IS012944 的这部分中。

对于浸在水中或埋在土壤中的钢结构, 腐蚀通常事实上是局部的, 而且腐蚀性级别是很难定义的。具体详见表 2 表 2 水和土壤的腐蚀分类

\begin{tabular}{|c|c|c|}
\hline 分类 & 环境 & 环境和结构的案例 \\
\hline Im1 & 淡水 & 河流上安装的设施, 水力发电站 \\
\hline Im2 & 海水或微咸水 & 没有阴极保护的浸入式结构(例如:港口 \\
& 区域, 如闸门、水闸或防波堤) \\
\hline Im3 & 土壤 & 埋地储罐、钢桩和钢管 \\
\hline Im4 & 海水或微咸水 & 带有阴极保护的浸入式结构(例如海上结构) \\
\hline & 注意: 腐蚀性类别Im1 和Im3, 阴极保护可与涂料体系进行相应的测试。 \\
\hline
\end{tabular}

\section{2 钢制结构表面处理}

\section{1 钢制结构表面预处理}

钢结构在涂装之前需要进行适当的表面处理. 去除钢材表面锈蚀产物等杂质, 如锈蚀、氧化皮、油污等杂质, 钢 制结构表面在涂装前的处理好坏程度. 直接关系到整个涂装体系的防腐性能和防护寿命。通过长期的实践证明. 许多 防护体系的提早失效. 其原因的 70-80\%以上是由于表明处理不良引起的。

钢结构的表面处理方式主要有抛丸、喷砂、手工和动力工具打磨、化学浸泡以及酸洗等方法。在实际施工过程中, 钢结构常采用抛丸和喷砂处理方法, 而部分补漆打磨采用局部手工及动力工具打磨处理方式。在完成表面处理后, 其 最终的表面处理状态按照 IS08501-88、GB/T8923-88 以及 SSPC 分为不同的等级, 表面处理的结果以比对板进行比较 检验。现将上述三个标准的表面处理状态对应关系如下表 3 ,

表 3 标准间表面状态对比

\begin{tabular}{|c|c|c|c|}
\hline 序号 & SPCC & GB / T $8923-2011$ & IS0-8501-2007 \\
\hline 1 & SP5 & Sa 3 & Sa 3 \\
\hline 2 & SP10 & Sa 2.5 & Sa 2.5 \\
\hline 3 & SP6 & Sa 2 & Sa 2 \\
\hline 4 & SP7 & Sa 1 & Sa 1 \\
\hline 5 & SP3 & St 3 & St $3 /$ St 3 \\
\hline 6 & SP2 & St 2 & St 3/St 2 \\
\hline
\end{tabular}

\section{2 钢制结构表面处理检查}

钢制结构表面处理完成后，主要的检查项目包括表面清洁度、表面粗䊅度、表面盐含量。

\section{2 .1 表面清洁度}

钢结构表面清洁度对油漆附着力以及外观质量有着重要影响, 当表面清洁度不足时会降低油漆附着力，过早地出现 的局部脱落以及表面凹凸不平等现象，因此在实际施工过程中，会在完成除锈后采用吸尘器等方式对设备表面进行一 次除尘处理，去除表面灰尘及颗粒，确保钢结构表面干净平整。具体的检测标准可根据 IS08502-3 或者 GB/T 18570-3 进行确定, 在施工过程中, 采用压敏粘带法来检测其表面灰尘是否满足要求, 一般钢结构在 3 级以内。具体检测标准 数值如下表 4 和图 1.

表 4 灰尘尺寸表

\begin{tabular}{|c|l|}
\hline 等级 & \multicolumn{1}{|c|}{ 灰尘微粒描述 } \\
\hline 0 & 用 10 倍放大镜开不见的微粒 \\
\hline 1 & 用10倍放大镜看见, 但用正常或者矫正视力不可见（通常微粒直径小于 $50 \mu \mathrm{m}$ ） \\
\hline 2 & 正常或矫正视力刚刚可见（通常微粒直径在 $50 \mu \mathrm{m}^{\sim} 100 \mu \mathrm{m}$ 间） \\
\hline 3 & 正常或者矫正视力清楚可见（通常微粒直径达到 $0.5 \mathrm{~mm}$ ） \\
\hline 4 & 微粒直径在 $0.5 \mathrm{~mm}$ 至 $2.5 \mathrm{~mm}$ 之间 \\
\hline 5 & 微粒直径大于 $2.5 \mathrm{~mm}$ \\
\hline
\end{tabular}


图 1 表面清洁度等级

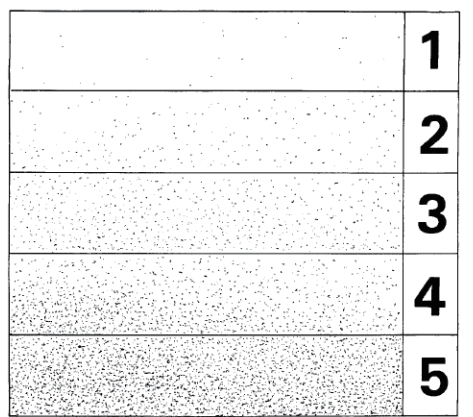

\section{2.2 表面粗粘度}

钢结构表面粗糙度是钢结构表面处理的关键，也是油漆防腐的关键控制点，粗糙的表面能增加钢结构表面与涂料 的接触面积, 提高其附着力, 但其粗粘度的大小需满足据设计、油漆产品以及标准规范要求, 且整个结构表面的粗粘 度应该均匀分布, 避免出现粗粘度分布不均现象, 造成涂料厚薄不均。另外粗糙度应避免出现过大或者过小的现象, 过小时会造成涂料吸附能力下降, 过早出现脱落, 防腐效果不佳; 过大时不能确保涂料能覆盖住粗糙度的波峰, 从而 造成波峰处漆膜过薄, 导致整体防腐性能下降。根据 API、ASME、ISO、IPS 等标准, 目前大部分钢结构粗糙度平均值 在 $30 \sim 80 \mu \mathrm{m}$ 之间, 但在实际施工过程中, 表面粗糙度难以把控, 其数值一般都会在 $100 \mu \mathrm{m}$ 左右, 施工现场通常采用 通过图片对比和针纹仪测量等方式进行测量。

因不同钢制结构所处环境不一致, 其漆膜厚度也不一样, 针对漆膜厚度要求薄的场所, 其喷漆厚度与喷砂工人的 作业水平有直接关系, 而石油等高腐蚀环境, 一般漆膜厚度在 $200 \mu \mathrm{m} \sim 00 \mu \mathrm{m}$ 之间, 在施工过程中只需要满足其表面 粗粘均匀性即可，因此综合来看，选用合理的磨料和相应的技术工人是关键。

另外表面粗粗粘度通常受以下因素影响:

1) 磨料的种类。石英砂、金刚砂、矿渣等;

2）磨料尺寸。通常尺寸在 1 2 $2 \mathrm{~mm}$ 之间;

3）喷砂角度、时长以及与钢结构表面的距离;

2.2. 3 表面盐含量

表面盐含量检测对钢结构防腐是非常关键的检查项，特别是针对旧钢结构再防腐。在试验和实际项目中都不难发现， 盐的对于设备腐蚀有重大影响, 以伊朗某项目为例, 在维护过程中发现, 凡是在含盐环境中的钢结构, 其腐蚀程度均 高于与无盐环境, 高盐环境腐蚀程度高于低盐环境。对于钢制设备和管道, 一旦出现防腐层破损, 其高盐环境会集中、 加速腐蚀破坏点, 从而出现穿孔、减薄、破损等现象, 最终出现大面积腐蚀。而且旧设备由于已运行一段时间, 其表 面盐含量通常会远高于新设备, 因此为确保防腐质量, 我们需要在油漆喷涂前采用软化水进行清洗, 直到表面盐含量 满足要求。具体顺序如下:

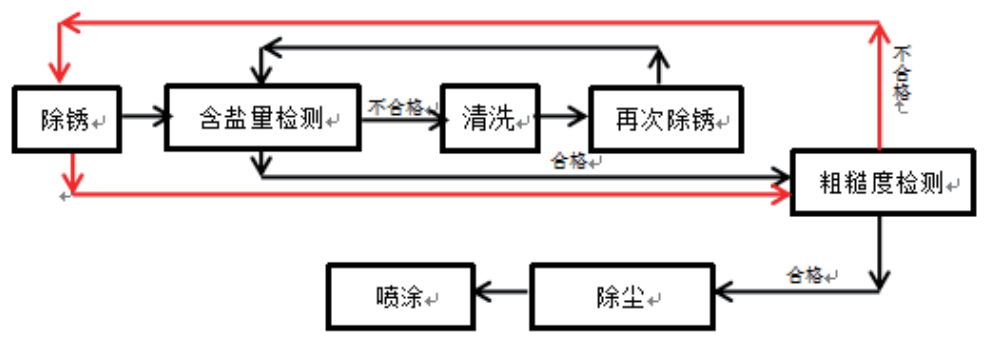

含盐量测试通常有三种方法, 包括擦拭法、贴片法及套管铁片法, 其原理为将钢制结构表面的盐等其他可溶性物 质溶于一定剂量的溶剂中便于测量，而钢制结构表面盐含量根据不通运行环境其接受值也不同。

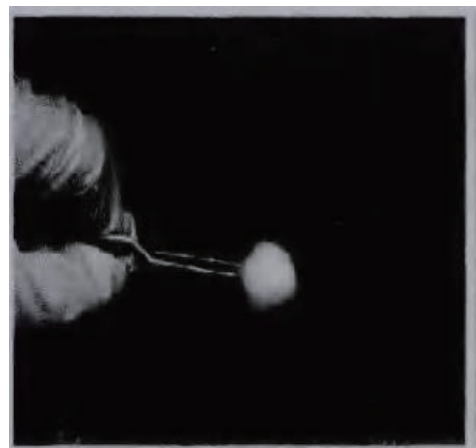

擦拭法

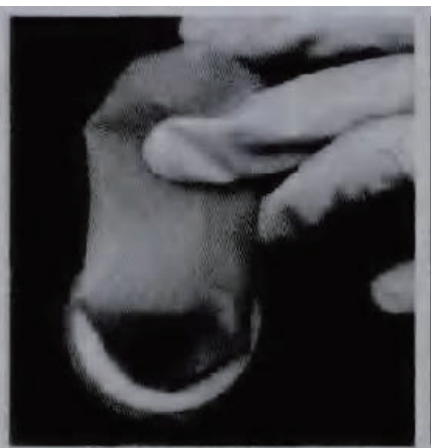

套管贴片法

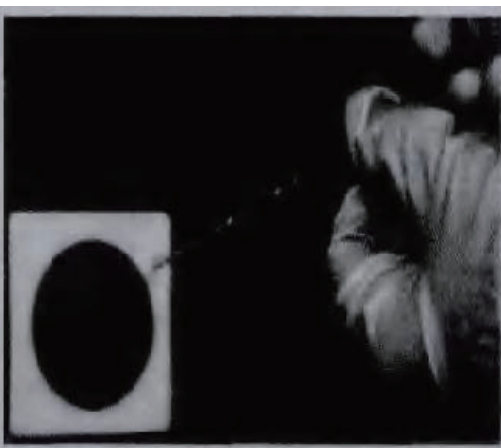

贴片法 
目前常用的方法是根据 IS080502-6 而制定的 Bresle 片测试表面盐污染, 其测试步骤见参考文件 - 表面盐污染测 试和计算方法。最终测试结果的导电率与单位重量的换算公式如下:

导电率 $\left(\mu \mathrm{S} . \mathrm{cm}^{-1}\right) \times 0.118$ = 单位重量 $(\mu \mathrm{g} . \mathrm{cm}-2)$; 此系数 0.118 为溶液取 3 毫升时的常量。

导电率单位 $\mu \mathrm{S} . \mathrm{cm}-1-$ - 即 microsiemens $\mathrm{cm}$ 微西门子厘米, 简称微西厘米;

单位重量 $\mu \mathrm{g} . \mathrm{cm}-2-----$ 微克每平方厘米

可以接受的盐污染:

目前还没有一个可接受盐污染水平的工业标准。它最终取决一系列因素, 包括使用涂料的种类、涂层的道数和厚度、 涂料体系的使用寿命以及所使用涂料的服务环境。

通常可以共同认可和接受的可接受盐污染标准是：对于使用在极端服务环境下的涂料, 如装载化学品的货舱, 仅 容忍较低的盐污染水平; 对于水线以上区域可接受较高的盐污染水平。然而, 必须共识和接受这样一个事实, 即任何 规定的最大可接受盐污染水平事实上是一定达到的。对于修船, 在规定一个人为的最大可接受盐污染水平方面, 绝对 没有这样的指标，比如小于 $1 \mu \mathrm{g} / \mathrm{cm}^{2}$ ，因为在先前已经腐蚀的表面是不可能达到的。

表 - 盐污染水平最大容许的总的可溶性盐水平

\begin{tabular}{|c|c|c|}
\hline $\begin{array}{l}\text { 盐污染水平 } \\
\left(\mu \mathrm{g} / \mathrm{cm}^{2}\right)\end{array}$ & $\begin{array}{l}\text { 盐污染水平 } \\
\left(\mathrm{mg} / \mathrm{m}^{2}\right)\end{array}$ & 内容 \\
\hline $210 \%$ m & (n) & $\begin{array}{l}\text { 最佳的水平 - 对使用在所有的环境包括浸水和大气暴露涂料 } \\
\text { 系统。仅能在新的结构上获得。适宜于所有的涂料系统。 }\end{array}$ \\
\hline 2 & 20 & $\begin{array}{l}\text { 可接受的水平 - NORSOK M-501, Rev4- - 《海洋工程处钢结构表面处理 } \\
\text { 和涂料保护》的要求。仅能在新的结构上获得。适宜于所有的涂料系统。 }\end{array}$ \\
\hline 2.5 & 25 & $\begin{array}{l}\text { 作为常规推荐首选的水平 - 适用于所有的环境包括在浸水和 } \\
\text { 大气暴露条件下, 包括海洋工程钢结构、石油储藏船等, 哪里 } \\
\text { 涂料施工条件是苛刻的, 设想延长到第一次维修的寿命。 }\end{array}$ \\
\hline 3 & 30 & $\begin{array}{l}\text { 规定在由“油船结构合作论坛”发表的《压载水 } \\
\text { 舱涂料系统和表面处理指导》的水平 }\end{array}$ \\
\hline 3.25 & 32.5 & 可接受的水平 - 引用美国NAVSEA对于压载水舱的《维护程序指导》。 \\
\hline 5 & 50 & $\begin{array}{l}\text { 可接受的水平 - 用于所有浸水和大气暴露的新的结构和维修, 包括 } \\
\text { 压载水舱、饮用水舱、灰水舱、海洋工程钢结构、石油储藏船等, } \\
\text { 哪里涂料施工条件是苛刻的, 设想延长到第一次维修的寿命。 }\end{array}$ \\
\hline 7.5 & 75 & $\begin{array}{l}\text { 可接受的水平 - 引用美国NAVSEA对于非浸 } \\
\text { 水的钢结构的《维护程序指导》 }\end{array}$ \\
\hline 10 & 100 & $\begin{array}{l}\text { 可接受的水平 - 引用SSPC - SP12/NACE5 SC - } 2 \text { 以下条 } \\
\text { 件, 适用于浸水和大气暴露的条件下的维修, 适用于表面处 } \\
\text { 理宽容性涂料在已生过锈的在大气环境下服务的钢 板、在 } \\
\text { 海水中航行船舶的压载舱、陆上玻璃钢储油罐维修系统 }\end{array}$ \\
\hline$>10$ & $>100$ & 超过极限的总的可溶性盐水平, 会导致降低涂料系统的性能。 \\
\hline
\end{tabular}

从上表能看出, 其表面处理含盐量可接受值低于 $10 \mu \mathrm{g} / \mathrm{cm}^{2}$

\section{3 钢制结构表面处理过程中容易忽略项}

2.3. 1 磨料选择

磨料应选择干燥、无油污的, 禁止使用潮湿、污染的磨料, 避免在喷砂除锈过程中加重钢制结构表面的污染程度。

2.3.2 压缩空气

在喷砂过程中使用空气压缩机作为气源, 需将空气出口接入空气净化器, 将气体中的水汽、油污清除, 避免在喷 砂过程中造成磨料污染和金属表面污染。

\section{3 .3 磨料循环使用}

磨料根据物理结构可以分为金属和非金属, 目前常用的金属为钢丸、钢砂等, 非金属为石英砂、金刚砂、铜矿和 铁矿渣等, 其通常作为一次性使用, 对于不锈钢材料, 所用磨料中不应含碳和氯离子, 但是在实际中除不锈钢处理外, 其他材料常将磨料混合循环使用。

对于循环使用的材料, 在使用过程中需要定期清理和更新混合配比, 清理磨料中的油污磨料和粒径过小的磨料, 控 制磨料的油污、电导率、干湿度和 PH 值, 混合磨料的配比和清洁度直接影响预处理后结构表面的状态、粗鋉度、清洁度。

\subsection{4 喷砂前钢结构表面清理}

实际作业中, 部分旧钢制结构表面滞留大量油污, 在喷砂过程中, 容易造成效率较低、磨料成本增加以及预处理 后表面清洁度不合格等现象，因此需要提前采用溶剂的方式进行清理。

23.5 喷砂后的除尘

由于在喷砂过程中出现大量粉尘, 在喷砂完成后容易造成钢结构表面堆积大量灰尘, 影响油漆喷涂质量, 造成油 漆脱落或者吸附不力, 因此需要提前用设备进行吸附处理, 但是在除尘过程中, 需要及时安排人员, 在保护措施到位 的情况下进行，避免因时间过长和人员出入，出现返锈（特别是高湿度时段）和二次污染。

2.3.6 喷砂后缺陷

通常在喷砂完成后, 会发现新缺陷, 这些缺陷导致钢结构表面粗糙度不一致, 会影响喷涂防腐质量和外观, 但由 
于钢结构表面需在 $4 \mathrm{~h}$ 内喷涂, 避免长时间放置出现返锈的情况, 因此需要及时处理, 在时间有限的情况下, 可以先覆 盖缺陷, 待底漆完成后再局部处理。

2.3.7 喷涂时间间隔

油漆时间间隔通常由油漆制造商根据产品不同而设置, 我们在油漆喷涂过程中需要根据说明书合理安排时间, 避 免时间间隔过短或者过长, 影响防腐质量。过短时造成层间凝固时间不足、硬度不够, 不能保持各自层的特性, 时间 过长时造成油漆太干以及表面过于光面, 后面油漆不能有效的附着, 形成层间空层和夹层, 容易脱落。 3 结论

3.1 不同环境对涂层要求区别很大, 漆膜厚度要求也不一样, 但是钢结构表面处理基本一致, 因此钢结构表面处理 是防腐施工的重点;

3.2 通过实验观察和实际施工作业, 在钢结构表面处理中, 表面含盐量与表面粗粘度、表面清洁度同等重要, 特别 是对于旧设备的再防腐施工，因此钢结构表面含盐量检测应作为旧设备防腐检测的监控重点。

\section{[参考文献]}

[1]史文婧.表面预处理对于钢结构防腐的重要性 [J].山东工业技术, 2014, 2 (7): 24-26.

[2] 金晓鸥. 防腐蚀涂装工程手册 [M]. 北京: 化学工业出版社, 2008.

[3] James R. Johnson. Soluble salts and specification-coatingsPro[Z]. May, 2008. 